\title{
Behçet's disease: a new target for anti-tumour necrosis factor treatment
}

\section{P P Sfikakis}

Ann Rheum Dis 2002;61(Suppl II):ii51-ii53

Behçet's disease is a multisystemic, chronic relapsing inflammatory disease classified among the vasculitides. Recurrent mucocutaneous lesions may be the only symptoms in mild cases, but articular, ocular, vascular, and/or gastrointestinal and central nervous system involvement may occur in most of the patients. Ocular disease is the most frequent cause of morbidity leading to blindness in $25 \%$ of those affected. The various non-specific immunosuppressive drugs, used either alone or in combinations, frequently fail to control inflammation or maintain remissions. The aetiology of Behçet's disease is unknown, however it is currently thought that a central pathogenetic role of tumour necrosis factor (TNF) in the inflammatory process is possible. Until June 2002, and according to published and anecdotal data, more than 80 patients from 10 different countries have received anti-TNF treatment.

The short-term effects of the anti-TNF monoclonal antibody infliximab have been reported in several case reports and small case series, whereas the effects of etanercept have been presented in recent conferences. Preliminary results strongly suggest that infliximab is remarkably effective in inducing short-term remission of almost all manifestations of the disease, including acute, sight threatening panuveitis. A double blind, placebo controlled, one month study on the efficacy of etanercept in suppressing the mucocutaneous manifestations of the disease showed beneficial results.

To date, significant side effects have not been reported. It seems that TNF block is an effective new treatment for patients with Behçet's disease. Whether such treatment is superior to the conventional therapeutic approaches in preventing relapses and progression of the disease remains to be determined by carefully controlled studies. At least three open, long term studies, including larger numbers of patients are currently being conducted.

$\mathrm{B}$ ehçet's disease is a chronic relapsing, multisystemic, inflammatory disorder. It affects male and female patients almost equally, although men have often more severe disease. The disease occurs endemically in the Middle East and Mediterranean regions, as well as in central and Far Eastern Asian countries including Iran, Iraq, Korea, China, and Japan. In contrast, the prevalence is very low in northern Europe and USA. Although recurrent mucocutaneous lesions may be the only symptoms in mild cases, ocular $(50 \%-70 \%)$, articular $(50 \%)$, vascular $(25 \%)$, gastrointestinal $(5 \%-10 \%)$, and central nervous system involvement $(5 \%-10 \%)$ may occur. Recurrent oral ulcers are often the first symptoms appearing in the early post-pubertal age. Ocular disease is the most frequent cause of morbidity leading to blindness $20 \%-25 \%$ of those affected..$^{12}$ The diagnosis of Behçet's disease is based on clinical criteria and no pathognomonic laboratory findings exist. Classification criteria used for patients participating in research protocols depend on the presence of recurrent oral ulceration, the hallmark of this disease, plus any two of the following: recurrent genital ulcerations, ocular lesions (anterior or posterior uveitis, or cells in vitreous or slit lamp examination, or retinal vasculitis), typical skin lesions, and a positive pathergy (skin hyperreactivity) test. ${ }^{3}$ Treatment remains largely symptomatic and empiric. Colchicine ${ }^{4}$ and thalidomide ${ }^{5}$ are effective for mucocutaneous manifestations. Combinations of corticosteroids and non-selective immunosuppressive drugs, including azathioprine, ${ }^{6}$ methotrexate, ${ }^{7}$ cyclosporine $\mathrm{A}^{89}{ }^{8}$ tacrolimus, ${ }^{10}$ chlorambucil, or cyclophosphamide ${ }^{79}$ are used when vital organs are involved. Interferon alfa is also an effective alternative treatment for the mucocutaneous manifestations ${ }^{11}$ and perhaps for ocular disease. ${ }^{12} 13$

Behçet's disease is characterised by spontaneous exacerbations and remissions, while the rate of symptoms' recurrence and healing time vary greatly among patients. Although the course of inflammation is classically intermittent, the disease can stabilise in a significant number of patients and become chronic in a given organ system. ${ }^{1}$ Therefore, the goal of management is to treat early to avoid recurrences and irreversible damage to the vital organs. Despite the chronic administration of intensive immunosuppressive treatment, however, permanent loss of vision resulting from multiple relapses of ocular inflammation occurs frequently. ${ }^{127}$

Inflammation in Behçet's disease is thought to be mediated by cytokines derived from $\mathrm{T}$ helper type 1 lymphocytes, including TNF. ${ }^{14}$ Increased numbers of monocytes and T lymphocytes expressing the gammadelta receptor that overproduce TNF are also found in patients with active disease. ${ }^{15} 16$ Increased levels of circulating TNF and soluble TNF receptors in the peripheral blood of patients with active disease, ${ }^{17}{ }^{18}$ as well as high levels of TNF in the aqueous humor from patients with Behçet's disease associated uveitis have been reported..$^{19}{ }^{20}$ A central pathogenetic role of TNF is further supported by the remarkably beneficial published results in Behçet's patients who have received the anti-TNF monoclonal antibody infliximab to date, ${ }^{21-26}$ including our five patients with sight threatening panuveitis. ${ }^{27}$ These results, as well as data on the effects of anti-TNF treatment in additional patients presented in recent conferences are summarised below.

\section{EFFECTS OF INFLIXIMAB}

A total of 11 cases of refractory to conventional treatment Behçet's disease, in which short-term anti-TNF treatment was given, were published during 2001. Infliximab at doses 3 $\mathrm{mg} / \mathrm{kg}$ (one patient), $5 \mathrm{mg} / \mathrm{kg}$ (nine patients), and $10 \mathrm{mg} / \mathrm{kg}$ (one patient) was used in all these cases. Duration of treatment varied from a single infusion to four infusions within a six month period. Infliximab administration was decided to treat severe mucocutaneous manifestations in two patients, ${ }^{21}{ }^{22}$ severe gastrointestinal involvement in three 
patients, ${ }^{23}{ }^{24}$ and sight threatening ocular relapse in six patients. ${ }^{25}{ }^{27}$ Combination immunosuppressive treatment had failed to induce remission in all these patients. A prompt improvement of longlasting orogenital ulcerations within two weeks after the infusion of infliximab was observed in the first two patients. Similarly, all gastrointestinal and extraintestinal symptoms, including synovitis and thrombophlebitis in one patient, improved dramatically or resolved completely within one to two weeks after infliximab administration. Finally, a single infusion of infliximab $(5 \mathrm{mg} / \mathrm{kg})$ was given to our five patients with relapsing panuveitis within 48 hours of relapse onset; remission of ocular inflammation was evident within the first 24 hours and complete suppression with a striking improvement of visual acuity was observed seven days after treatment in all. At the onset of the ocular relapse concomitant oral ulcers were present in two patients and oligoarthritis developed in a third patient. Oral ulcers healed by day 2 after infliximab administration in both patients, while the oligoarthritis subsided within four days after treatment. ${ }^{27}$ Identical results using $5 \mathrm{mg} / \mathrm{kg}$ of infliximab were subsequently reported in one Spanish patient with panuveitis. ${ }^{25}$ No adverse effects were noted in any of those 11 patients and a sustained response during the next months was observed in most of them (references ${ }^{21-25}$ and our unpublished observations). Beneficial preliminary results in two groups of patients in Japan (personal communication) and the Netherlands (personal communication) have also been obtained with infliximab for the treatment of ocular disease.

Eleven additional patients with Behçet's disease refractory to previous immunosuppressive treatment, including nine patients with severe ocular involvement, one with cerebral vasculitis, and one with severe mucocutaneous manifestations and arthritis, were presented by five different groups at the 10th International Conference on Behçet's disease held in Berlin in June 2002. These patients, except for the last patient in whom remission could not be achieved, responded well to short-term infliximab treatment without side effects. We are currently conducting an open study on the long term effects of infliximab on visual outcome and extraocular manifestations in patients with active disease who failed to respond to conventional immunosuppressive treatment. Similar studies are underway in Turkey and Japan. Two of our patients have completed 20 months and 16 months, respectively, of continuous treatment with infliximab (Remicade, $5 \mathrm{mg} / \mathrm{kg}$ intravenously, at days 1, 30, and every two months thereafter); both patients remain in complete remission using minimal daily doses of prednisone and cyclosporine A, respectively. We have not observed any adverse effects, except for low titres of serum antinuclear and anti-dsDNA antibodies in one patient, detected nine months after treatment with infliximab had begun; no lupus related clinical signs have been observed during the next 11 months of continuous treatment.

\section{EFFECTS OF ETANERCEPT}

Up to June 2002 there was no published evidence on the use of etanercept in Behçet's disease. However, an elegant study of four week duration was recently completed in Istanbul by the group led by Hassan Yazici and the results were presented at the 10th International Conference on Behçet's disease. This double blind, placebo controlled study examined the efficacy of etanercept in suppressing the mucocutaneous manifestations of the disease. Forty male patients, all with mainly mucocutaneous disease, after a four week wash out of all medications were randomised to receive either etanercept 25 mg subcutaneoulsy, twice a week, or placebo. Significant beneficial effects of etanercept were seen in the numbers of oral ulcers, nodular and papulopustular skin lesions, as well as in arthritis episodes. These effects were observed by the end of the first week of treatment. Etanercept treated patients had a $40 \%$ chance of remaining free of oral ulcers at the end of treatment, compared with an only $5 \%$ chance in the placebo group. Etanercept treatment resulted in significant decreases of erythrocyte sedimentation rate and $\mathrm{C}$ reactive protein serum levels. However, recurrence of symptoms were noted in some patients three months after etanercept was stopped.

In an additional study performed by the same group of investigators, the effect of etanercept ( $25 \mathrm{mg}$ subcutaneously, twice a week for six months) on visual acuity in 10 patients with refractory uveitis was examined. All patients had severe ocular inflammation despite combination treatment with corticosteroids, azathioprine, and cyclosporine A. Addition of etanercept in their previous treatment had a beneficial effect in maintaining visual acuity with a concomitant decrease of corticosteroid dose. This effect lasted during the whole treatment period but was not sustained in the six month post-treatment follow up.

\section{PRELIMINARY CONCLUSIONS}

Among anti-TNF agents, infliximab and etanercept have recently started to be used in the treatment of Behçet's disease. A survey conducted at the 10th International conference on Behçet's disease revealed that among experienced physicians specialised in this disease $38 \%$ has already tried anti-TNF block in their patients. Given the variable and limited success of other interventions, and although the available information only derives from the small number of patients treated in the short-term so far, such treatment seems to be an effective new therapeutic approach for patients with refractory disease.

Data that continue to accumulate strongly suggest that infliximab administration leads to rapid and effective suppression of almost all Behçet's disease manifestations, at least in the short-term. Regarding patients with acute ocular inflammation a rapid therapeutic effect is critical to prevent the development of fixed retinal lesions that cause permanent visual impairment in the long term course of this relapsing condition. We have confirmed our initial results on sight threatening panuveitis ${ }^{27}$ in more than 15 Greek patients to date. Such rapid effects are usually not observed with the current therapeutic approach, using large doses of non-selective immunosuppressive agents. ${ }^{28}$ A possibility that the efficacy of infliximab is superior to etanercept in Behçet's disease may exist, although this has not been confirmed. As it was established recently, differences existing in the mode of action between these two anti-TNF agents account for their differential efficacy in inhibiting the inflammation in Crohn's disease. ${ }^{29}$ Significant side effects with the use of these agents in Behçet's disease have not been reported. On the other hand, few patients have received long term treatment thus far. Whether such treatment is superior to the conventional therapeutic approaches in preventing relapses and progression of the disease remains to be determined by carefully controlled studies. Because a delay of treating the ocular disease will negatively affect the long term outcome of visual acuity, a comparison with placebo in such patients is no longer justified. The results of at least three open, ongoing studies examining the long term efficacy and safety of TNF block in Behçet's disease are forthcoming.

\section{Author's affiliations}

P P Sfikakis, First Department of Propaedeutic Medicine, Laikon Hospital, Athens University Medical School, Athens, Greece Correspondence to: Dr P P Sfikakis, 3 Amaryllidos Str, 154 52, Athens, Greece; psfikakis@med.voa.gr

\section{REFERENCES}

1 Kaklamani VG, Vaiopoulos G, Kaklamanis PG. Behçet's disease. Semin Arthritis Rheum 1998;27:197-217.

2 Yazici H. Behçet's syndrome: Where do we stand? Am J Med 2002; 1 12:75-6. 
3 International Study Group for Behçet's disease. Criteria for diagnosis of Behçet's disease. Lancet 1990;335:1078-80.

4 Yardacul S, Mat C, Tuzun Y, et al. A double-blind trial of colchicine in Behçet's syndrome. Arthritis Rheum 2001;44:2686-92.

5 Hamaryudan V, Mat C, Saip S, et al. Thalidomide in the treatment of the mucocutaneous lesions of the Behçet's syndrome. A randomized, double blind, placebo controlled trial. Ann Intern Med 1998; 128:443-50

6 Yazici $\mathbf{H}$, Pazarli H, Barnes CG, et al. A controlled trial of azathioprine in Behçet's syndrome. N Engl J Med 1990;322:281-5.

7 Kaklamani VG, Kaklamanis PG. Treatment of Behçet's disease: an update. Semin Arthritis Rheum 2001;30:299-312.

8 Masuda K, Nakajima A, Urayama A, Nakae K, Kogure M, Inaba G. Double-masked trial of cyclosporin versus colchicine and long-term open study of cyclosporin in Behçet's disease. Lancet 1989;i:1093-6.

9 Ozyazgan Y, Yurdakul S, Yazici H, et al. Low dose cyclosporin-A versus pulsed cyclophospamide Behçet's syndrome: a single-masked trial. $\mathrm{Br} J$ Ophthalmol 1992;76:241-3.

10 Ishioka $M$, Ohno S, Nakamura S, et al. FK506 treatment of noninfectious uveitis. Am J Ophthalmol 1994;1 18:723-9.

11 Alpsoy E, Durusoy C, Yilmaz E, et al. Interferon alfa-2a in the treatment of Behçet's disease: a randomized placebo-controlled and double-blind study. Arch Dermatol 2002;138:467-71

12 Kotter I, Eckstein AK, Stubiger N, Zierhut M. Treatment of ocular symptoms of Behçet's disease with Interferon alfa-2a: a pilot study. $\mathrm{Br} J$ Ophthalmol 1998;82:488-94.

13 Wechsler B, Bodaghi B, Huong DL, et al. Efficacy of Interferon alfa-2a in severe and refractory uveitis associated with Behçet's disease. Ocul Immunol Inflamm 2000;8:293-301.

14 Gul A. Behçet's disease : an update on the pathogenesis. Clin Exp Rheumatol 2001;19 (suppl 24):S6-12.

15 Yamashita N, Kaneoka H, Kaneko S, et al. Role of gammadelta T lymphocytes in the development of Behçet's disease. Clin Exp Immuno 1997; 107:241-7.

16 Mege JL, Dilsen N, Sanguedolce V, et al. Overproduction of monocyte-derive TNF-a, IL-6, IL-8 and increased neutrophil superoxide generation in Behçet's disease. A comparative study with familial Mediterranean fever and healthy subjects. J Rheumatol 1993;20:1544-9.
17 Turan B, Gallati H, Erdi H, Gurler A, Michel BA, Villiger PM. Systemic levels of the T cell regulatory cytokines IL-10 and IL-12 in Behçet's disease; soluble TNFR-75 as a biological marker of disease activity. J Rheumatol 1997;24:128-32.

18 Evereklioglu C, Er H, Turkoz Y, Cekmen M. Serum levels of TNF-a, sIL-2R, OL-6, and IL-8 are increased and associated with elevated lipid peroxidation in patients with Behçet's disease. Mediators Inflamm 2002;1 1:87-93

19 Chronopoulou H, Tzavara V, Oikonomopoulos N, et al. High levels of TNF-a in the in the aqueous humor in patients suffering from Adamantiades-Behçet's disease: preliminary results. Invest Ophthalmol Vis Sci 2001;42 (suppl 4):S708

20 Santos Lacomba M, Marcos Martin C, Gallardo Galera JM, et al. Aqueous humor and serum tumor necrosis factor-alpha in clinical uveitis. Ophthalmic Res 2001;33:251-5.

21 Goossens PH, Verburg RJ, Breedveld FC. Remission of Behçet's syndrome with tumour necrosis factor a blocking therapy. Ann Rheum Dis 2001;60:637.

22 Robertson LP, Hickling P. Treatment of recalcitrant orogenital ulceration of Behçet's syndrome with Infliximab. Rheumatology 2001;40:473-4.

23 Hassard PV, Binder SW, Nelson V, Vasiliauskas EA. Anti-tumor necrosis factor monoclonal antibody therapy for gastrointestinal Behçet's disease: a case report. Gastroenterology 2001;120:995-9.

24 Travis SP, SP, Czajkowski M, McGovern DP, Watson RG, Bell AL. Treatment of intestinal Behçet's syndrome with chimeric tumor necrosis factor alpha antibody. Gut 2001;49:725-8.

25 Munoz-Fernandez S, Hidalgo V, Fernandez-Melon J, Schlincker A, Martin-Mola E. Effect of infliximab on threatening panuveitis in Behçet's disease. Lancet 2001;358:1644.

26 Rosenbaum M, Rosner I, Portnoy E. Remission of Behçet's syndrome with TNFa blocking treatment. Ann Rheum Dis 2002;61:283-4.

27 Sfikakis PP, Theodossiadis PG, Katsiari CG, Kaklamanis P, Markomichelakis NN. Effect of infliximab on sight-threatening panuveitis in Behcet's disease. Lancet 2001;358:295-6.

28 Nussenblatt RB. Uveitis in Behçet's disease. Int Rev Immunol 1997; 14:67-9.

29 van Deventer SJH. Transmembrane TNF-a, induction of apoptosis, and the efficacy of TNF-targeting therapies in Crohn's disease. Gastroenterology 2001;121:1242-5. 